\title{
Stochastic Modeling Of Spatial Heterogeneities In Microstructures
}

Dr. Asim Tewari*

*Senior Research Scientist, General Motors India Science Lab, Units 1-8, 3rd Floor, Creator Building, International Tech Park, Whitefield Road, Bangalore - 560066, INDIA

Composites and many advance-engineering alloys contain two or more phases, with one being a continuous phase and the other being discrete. Very often the spatial distribution of the discrete phase is not homogeneous throughout the microstructure. The origin of these spatial heterogeneities can be traced back to their fabrication route (casting, rolling, etc.) or inherent interactions (repulsion or attractions) among the phases. These spatial heterogeneities manifest themselves in the form of clusters, streaks or regions of depletion of the discrete phase. The properties of such materials are strong functions of these microstructural heterogeneities [1,2] and require quantitative description to better understand their response. Hence, there is a need to study, quantify and model these microstructural attributes.

In this article a new morphological point operation based methodology has been developed which would objectively identify and quantify such heterogeneities. This provides the size, orientation and density of clusters and groups together the second phase particles that belong to same cluster. The technique has been applied through digital morphology to second phase particles in an aluminum alloy sheets processed through continuous casting and compared with results from sheets processed through direct chill casting. The analysis shows streaks of second phase clusters with preferred 
orientations in the continuous cast sheets. This is shown in the figure 1 and 2 , where the original binary image is compared with the image after cluster analysis. The clusters on the sheet microstructure have been modeled by the union of two point processes [3] and have been show to have good correspondence with the real structure.

References

[1] L. Mishnaevsky et al.,Comp. Sc. Tech. 64 (Issue 12) (2004) 1805.

[2] M. Slamovaa et al., Mat. Char. 49 (Issue 3) (2002) 231.

[3] Dietrich Stoyan et al., Stochastic Geometry and Its Applications, 2nd ed., John Wiley \& Sons, 1996
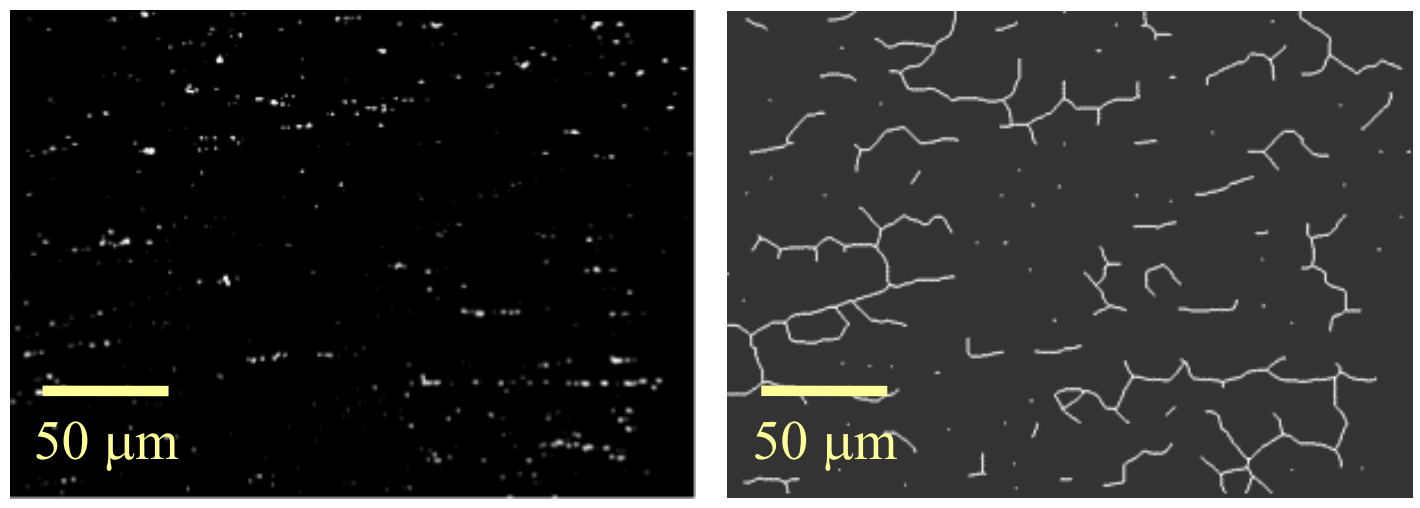

Figure 1: Binary image of continuous cast sheet before and after cluster analysis
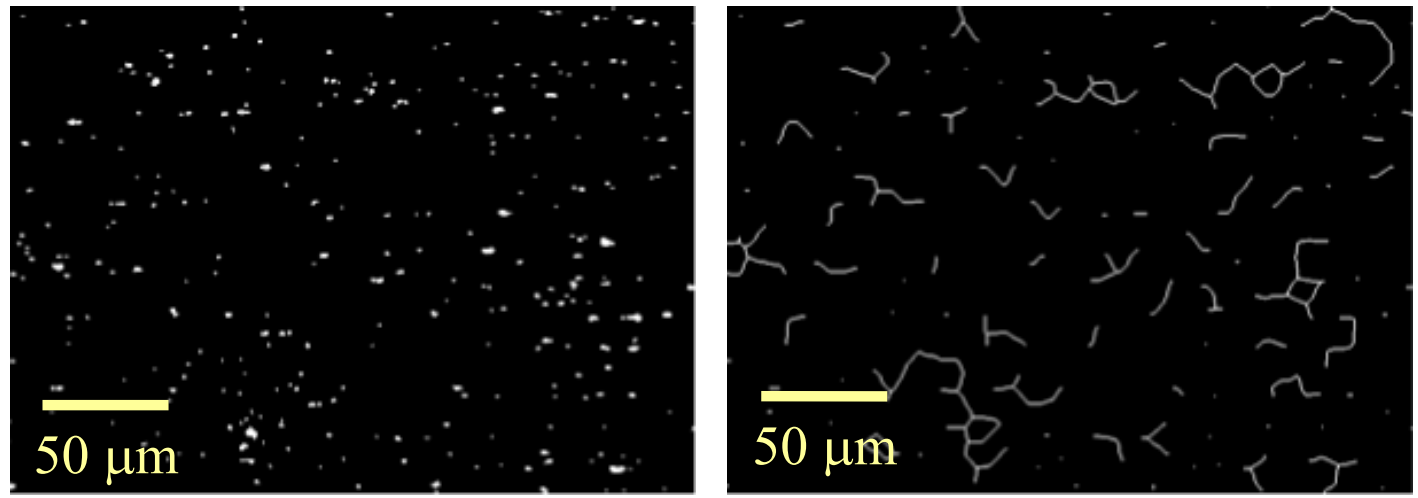

Figure 2: Binary image of direct chill cast sheet before and after cluster analysis 